\title{
Analysis Of Multi-energy Complementary Integration Optimization Technology
}

\author{
Xiaohong He, Lijun Zheng \\ Huadian Electric Power Research Institute Co., LTD., Hangzhou 310030, China
}

\begin{abstract}
This paper studies ways to improve the capacity of renewable energy to reduce the adverse effects of renewable energy generation on the power grid system, improve energy efficiency, and implement comprehensive complementary utilization of multiple energy sources. According to different resource conditions and energy demands, the multi-energy complementary systems are constructed through comprehensive energy management and collaborative optimization control. On the basis of summarizing the technical routes of multi-energy complementary system at home and abroad, the key technologies of multienergy complementary were discussed, including various power characteristics, complementary ways and future research directions. It provides a reference for the development and application of multi-energy complementary system
\end{abstract}

\section{Introduction}

In recent years, China's renewable energy power generation has made great progress, and hydropower installed capacity and wind power grid-connected capacity are among the highest in the world. However, in some areas, the capacity of renewable energy consumption market is limited, and the problem of abandoning wind, light and water is becoming increasingly prominent. In addition, renewable energy has intermittent, random and volatility, which adversely affects the shape and operation characteristics of the power grid. It makes the power system frequency modulation and voltage regulation capability decrease, and the anti-disturbance capability is insufficient, which brings pressure on the safe and stable operation of the system.

In order to improve the renewable energy consumption capacity and the overall efficiency of energy system, adapt to the transition trend of energy supply mode to green, efficient and close to users, China proposes to implement multi-energy complementary system integration optimization project. It mainly conducts research on power supply characteristics and complementary methods, system design, integration optimization, etc., and deeply explores multi-energy complementary implementation schemes and key technologies.

\section{Overview of the technical route of multi-energy complementary systems}

Countries such as the United States, Europe, and Japan actively promote smart grid construction on the basis of distributed generation, providing a dynamic platform for access to various distributed energy sources. It uses renewable energy such as small and medium-sized hydropower resources and biomass resources to recycle various waste energy sources and increase electricity and other energy supplies. And through the energy ladder utilization system, renewable energy system and resource comprehensive utilization system in the user terminal market, improve energy utilization efficiency, reduce pollutant emissions, and optimize energy structure.

In 2001, the United States proposed the Integrated Energy System (IES) development plan to increase the proportion of clean energy supply and utilization, promote the promotion and application of distributed energy and cogeneration technologies, and develop micro-based on this basis. Power grid and smart grid.

In 2009, the EU Renewable Energy Directive came into effect and became the core and driving force of the EU's clean energy policy. Member states formulated and implemented the National Renewable Energy Action Plan in accordance with the requirements of the Directive. Germany implements the optimization and coordination of energy systems from the perspective of the entire supply chain of energy and the entire industry chain. The UK is more focused on the integration between energy systems and communication systems. In order to achieve the goal of $100 \%$ renewable energy in 2050, Denmark places special emphasis on power, natural gas and heating fusion technologies [1].

Japan is mainly engaged in the research and demonstration of smart community technology, and proposes a comprehensive energy system for the community. Based on the traditional energy supply system, build integrated energy system solutions (including electricity, gas, heat, renewable energy, etc.), 
and build a hydrogen energy supply network covering the whole society to achieve transportation, water supply, information and medical systems. Integrated integration.

China's multi-energy complementary integration optimization demonstration project is a systematic project that uses multiple energy sources to complement each other to achieve a virtuous cycle according to different resource conditions and energy use objects. The first batch of multi-energy complementary integration optimization demonstration engineering technology routes mainly include terminal integrated energy supply system and landscape energy storage system.

The terminal integrated energy supply system is designed to meet the needs of end users for power, heat, cold, gas, etc., and realize multi-energy collaborative supply and energy through natural gas multi-connection, distributed renewable energy and smart micro-grid. Comprehensive cascade utilization.

The multi-energy complementary system of scenery, water and fire storage utilizes the combined advantages of wind energy, solar energy, water energy, coal, natural gas and other resources in a large-scale comprehensive energy base, and adds large-scale energy storage equipment, which can not only exert the rapid adjustment capability of hydropower generation, but also supplement the active output of photovoltaic power stations. To improve the quality of photovoltaic power, it is also possible to provide photovoltaic power generation with hydropower generation, to provide a relatively stable and reliable power supply for the system, to promote the delivery of renewable energy, and to reduce the phenomenon of abandoned wind and light.

\section{Multi-energy complementation of various power supply features and complementary forms}

Multi-energy complementary systems usually include thermal power (including gas turbine), wind power, solar power (photovoltaic), hydropower, pumped storage and other types of power supply[2]. As a conventional schedulable power source, thermal power can be adjusted to generate a certain peak amplitude, and the output speed is slow. The gas turbine unit starts and stops quickly, and can be used as a backup and peaking power supply for the power system, and mainly bears the peak load of the power system. The effective capacity of renewable energy such as wind energy and solar power generation is almost zero, and the output changes frequently and uncontrollable. Hydropower stations with regulating capacity can use their reservoir water storage regulation, and wind power and photoelectric compensation operation to exert greater capacity benefits. The pumped storage power station can undertake the rapid response capacity and peak shaving task of the grid system, and improve the ability of the grid to accept wind power and photo electricity.

The complementary of multiple energy sources refers to the complementary utilization and redistribution of energy supply from a time perspective according to energy fluctuation characteristics and regulation capabilities, in order to improve the utilization of wind and light, improve the reliability of power output and the ability of the power grid to accept renewable energy. Research in this field mainly focuses on system modeling and simulation, optimization configuration, control strategy, and active power output characteristics. By using the complementary coefficient to combine the subsystem evaluation with the joint power generation system evaluation, an index evaluation system for the complementary characteristics of landscape water is constructed [3]. The research on the power generation operation characteristics shows that the active power output characteristics of the power generation system can maintain good system load. Tracking, there is strong complementarity in the time scale of the day.

Thermal complementation refers to the introduction of different thermal energy into the appropriate position in the thermodynamic cycle according to the principle of "temperature-to-port, cascade utilization", improving energy conversion efficiency and enhancing time complementary effect. Solar fossil fuel thermal complementary combined cycle power generation system has received extensive attention due to high hotspot conversion efficiency and thermal complementation diversity. Through the research on the integration scheme of tower solar energy and conventional coal-fired power plants [4], the complementary system has the highest photoelectric conversion efficiency of $16.97 \%$, and the cost is lower than the existing single solar thermal power generation system. In addition, the form of thermal complementation may also be that solar energy is complementary to biomass or geothermal energy, or that coal power is complementary to wind and light. In areas with rich geothermal energy and biomass resources, it is possible to build a combined cooling and heating system with solar energy to achieve full complementarity of renewable energy.

Multi-energy thermo-chemical complementary technology refers to the selection of a suitable endothermic chemical reaction to convert thermal energy into fuel chemical energy, improve energy conversion efficiency, and achieve renewable energy storage and transport. The technology is currently in the basic research stage. For example, medium-low temperature solar-powered methanol reforming reaction thermochemical power generation technology, landscape-hydrogen production hybrid technology. The literature [5] verified the effectiveness of the control strategy of wind power/hydrogen production/fuel cell/super capacitor hybrid system by simulation results of different operation modes.

\section{Analysis of key technologies of multi- energy complementary systems}

\subsection{Distributed Energy Generation Technology}

Multi-energy complementary power generation systems involve a variety of different technologies. A new generation of distributed energy generation technology is 
the most important technology. It mainly includes variable-speed constant-frequency wind power generation technology, large-scale photovoltaic power generation and solar thermal power generation technology, micro gas turbine power generation technology, advanced heat pump technology with regional thermal energy taste regulation, and low-cost commercial fuel cells. Research and development of key technologies and equipment such as energy conversion stations, energy hubs, smart energy and metering equipment at the customer end, and transportation networks based on smart electric vehicles are also receiving more attention.

In multi-energy complementary systems, the matching between multiple energy sources and multiple loads and the performance of variable operating conditions are the most important, which are major characteristics distinguishing from traditional distributed generation systems. Therefore, performance indicators such as overall energy utilization, power generation efficiency, and comprehensive energy conservation are particularly important. The parameters of unit and load are obtained by analyzing the annual load changes of multiple users in the system. The configuration schemes of the unit are optimized and analyzed to maximize energy efficiency. Therefore, high-precision multi-load prediction technology and multi-energy deep coupling utilization technology are key technologies for multienergy comprehensive optimization, which will be the main research direction in the future.

\subsection{Energy storage technology and energy storage configuration strategy}

Energy storage technology is the core foundation of multi-energy complementary systems to solve the mismatch between generating power and load power, the mismatch between response times of different types of power supplies. Energy storage in multi-energy complementary systems include power storage, such as pumped storage, compressed air storage, battery storage. In addition, energy storage technologies include storage and conversion of electrical energy and other forms of energy, such as electrochemical energy storage, heat storage, hydrogen storage, and electric vehicles. The coupling of multiple energy networks such as power grids, natural gas pipeline networks, cold and heat networks and transportation networks is achieved by the application of various energy storage methods.

The higher system cost performance is reached by selecting different characteristic energy storage devices in different multi-energy complementary systems. Optimal system energy efficiency is achieved by selecting energy storage units with different power and capacity. Energy efficiency and operating costs are optimized in energy management systems. The research and development key material, manufacturing processes and the improvement of energy conversion efficiency are the difficulties in the development of energy storage technology. The important parameters of the energy storage system are high capacity, low cost, long life cycle.

\subsection{Integrated energy management system}

The main management objects of integrated energy management include user load, distributed power supply, and transaction center. The energy flow is regulated by information flow to ensure efficient and stable operation of multi-energy complementary systems. For complex multi-energy complementary systems, through the establishment of a system platform for analytical processing and global optimization management, the core modules include forecasting, analysis and decisionmaking links, grid, renewable energy, non-renewable energy, energy storage systems, and various energy loads. In order to carry out organic integration, a reasonable conversion plan for energy output and different forms of energy at the energy supply end is formulated, and multi-dimensional comprehensive decision making is carried out $[6,7]$.

At present, the research on integrated energy management at home and abroad is still in its infancy, and there is still no systematic basic theory and mature system application. In [8], through the research on the operation control and energy management technology of the microgrid with multi-voltage source micro-source networking, it is shown that the unit start-stop phase, the intra-day economic optimization scheduling phase and the real-time adjustment phase of the scheduling plan are taken respectively. Different management strategies enable the system to operate safely and economically. The architecture of the self-disciplined smart grid energy management system is that multiple distributed centralized energy management subsystems form interconnection through two-way communication to realize multi-energy stream collaborative optimization management. The research in the next few years will focus on multi-energy flow real-time modeling and state estimation, multi-energy flow multi-time scale safety analysis and safety control, multi-energy flow mixed time scale optimization scheduling and energy management system construction, development and verification.

\subsection{Collaborative optimization control technology}

The multi-energy complementary system optimization control system can perform multi-energy complementary and optimal scheduling for various distributed energy systems based on load forecasting, distributed energy generation prediction, electricity price and gas price. Domestic research scholars proposed multi-power coordinated control technology and strategy, and verified the feasibility of the control strategy and the effectiveness of the system software. Literature [9-11] proposed centralized management and hierarchical/hierarchical control ideas, establishing local control, centralized control, and distribution network scheduling layers, through multi-energy system planning, 
intelligent regulation, collaborative control and interaction, comprehensive evaluation, and system information security. Research on key technologies such as communication and energy trading and business service operation mode, creates a collaborative optimization control software system architecture, support decentralized decision-making and centralized coordination, and realize coordinated control of internal and external pluripotent complementary systems.

In addition, related technologies of multi-energy complementary systems include large-capacity longdistance transmission technology, advanced power electronics technology, reliable and secure communication technology and standardization technology. The research will effectively ensure that the multi-energy complementary system outputs highquality electric energy, realizes the coordinated cooperation between the distributed power supply and the existing distribution network electrical equipment, and ensures the safe operation of the distribution network.

\section{Summary and outlook}

According to different resource conditions and energy demand, through integrated energy management and collaborative optimization control, a multi-energy complementary integrated optimization system can be built to improve the capacity of renewable energy consumption, achieve energy conservation and emission reduction targets, and promote the transformation of energy structure. Research institutions at home and abroad design multi-energy complementary energy systems according to different resource conditions and energy demand, adapt to local conditions, effectively improve the utilization efficiency of integrated energy systems, and ensure energy supply security.

In the future, based on the development of advanced distributed energy generation technology, we will pay more attention to the research of integrated energy management and multi-energy collaborative control technology, and pay attention to the energy storage system and multi-energy complementary in the development of large-scale and low-cost energy storage systems. The system adapts to ensure that the multienergy supply is stable, safe and reliable, and multienergy complementary and optimal scheduling for various power generation systems to maximize energy utilization efficiency.

\section{References}

1. Jianzhong,Wu.Drivers and State-of-the-art of Integrated Energy Systems in Europe[J]. Automation of Electric Power Systems,2016,40(05):1-7.A. Mecke, I. Lee, J.R. Baker jr., M.M. Banaszak Holl, B.G. Orr, Eur. Phys. J. E 14, 7 (2004)

2. Sheliang Wang,Li Feng,Pin Zhang,etc.The Hybrid of Multiple Energy Promotes New Energy
Development[J].Northwest

Hydropower,2014(06):78-82.

3. 3..Lin Ye,Xiaoxu Qu,Yanxiang Me,etc. Analysis on Intraday Operation Characteristics of Hybrid Windsolar-hydro Power Generation System[J]. Automation of Electric Power Systems, 2018,42(04):158-164.

4. Xiaohui Yu. Thermodynamic Performance Analysis of Solar Power Tower Aided Coal-fired Power Generation System[D].North China Electric Power University(Bei jing),2017.

5. Guowei Cai,Chong Chen,Lingguo Kong,etc. Control of Hybrid System of Wind/Hydrogen/Fuel Cell/Supercapacitor[J]. Transactions of China Electrotechnical Society, 2017,32(17):84-94.

6. Chong Chen. Research on Modeling and Gridconnected Control of Integrated System of Wind Power/hydroge/fuel Cell[D]. Northeast Electric Power University,2017.

7. Di Zhong,Qiming Li,Xian Zhou,etc. Research status and development trends for key technologies of multi-energy complementary comprehensive utilization system $[\mathrm{J}]$. Thermal Power Generation,2018,47(02):1-5+55.

8. Jianxiao Wang,Haiwang Zhong,Ziming Ma,Qing Xia,Chongqing Kang. Review and prospect of integrated demand response in the multi-energy system[J]. Applied Energy,2017,202.

9. Wei Bao. Research on Control and Energy Management Strategy of Micro-grid Composed of Multi Voltage Source Type Micro-sources[D]. China Electric Power Research Institute,2014.

10. Qian Ai,Ran Hao. Key Technologies and Challenges for Multi-energy Complementarity and Optimization of Integrated Energy System[J]. Automation of Electric Power Systems, 2018,42(04):2-10+46.

11. Ran Hao,Qian Ai,Yuchao Zhu,etc. Hierarchical optimal dispatch based on energy hub for regional integrated energy system[J]. Electric Power Automation Equipment,2017,37(06):171-178. 